\title{
Recommended laboratory and field studies ahead of future Mercury exploration
}

\author{
A White Paper in support of the 2023-2032 Planetary Science and Astrobiology Decadal \\ Survey
}

Lead Author: Kathleen E. Vander Kaaden, Phone: 281-244-5020, Affiliation: Jacobs, JETS Contract, NASA Johnson Space Center (JSC), Kathleen.e.vanderkaaden@nasa.gov

Co-Authors: Ronald J. Vervack Jr. (Johns Hopkins Applied Physics Laboratory), Elizabeth Rampe (NASA JSC), Francis M. McCubbin (NASA JSC), Christian Klimczak (University of Georgia), Christopher J. Cline II (Jacobs, JETS Contract, NASA JSC), Paul K. Byrne (North Carolina State University), Brendan A. Anzures (Brown University)

\section{Endorsers:}

Zoë Wilbur (University of Arizona), Arya Udry (University of Nevada Las Vegas), Michelle Thompson (Purdue University), Jay Thompson (Jacobs/NASA JSC), Soumya Ray (Arizona State University), Stephen Parman (Brown University), Marc Fries (NASA JSC), Stephen Elardo (University of Florida), Nicholas Dygert (University of Tennessee), Chuanfei Dong (Princeton University), Jessica Barnes (University of Arizona), Rosalind Armytage (Jacobs/NASA JSC) 


\subsection{INTRODUCTION}

Data from Mariner 10, MESSENGER, and ground-based telescopic observations have facilitated great advancements towards understanding the geochemistry, geology, internal structure, and space environment (exosphere/magnetosphere) of Mercury. Future exploration efforts by BepiColombo, landed science [1], and sample return [2] will revolutionize our understanding of this planet. However, to enhance the scientific return of past, present, and future data collection at Mercury, it is imperative that laboratory and field-based studies be conducted over the next decade. This white paper gives examples of laboratory and field studies, including instrument testing, which should take place over the next decade to facilitate the interpretation of data and preparation of future missions to the inner-most planet. By no means are the topics in this paper exhaustive, but rather are meant to demonstrate the immense amount of work that needs to be completed ahead of, or in conjunction with, current and future exploration efforts of Mercury. This work would amplify the benefits from additional data obtained from the planet's surface to the fullest extent possible, and to aid in decision making of future instrument payloads. We have chosen to focus our inputs here on geochemistry, geology, and space environment related efforts, but we emphasize that extensive research in the areas of geophysics and other geosciences-related disciplines will benefit from additional studies.

\subsection{GEOCHEMISTRY RELATED STUDIES}

We currently do not have any recognized samples from Mercury in our astromaterials collections. Additionally, we only have geochemical data from the MESSENGER mission for the surface of the planet, with the highest-resolution data available for the northern hemisphere. Therefore, much of the geochemical nature of Mercury, including its differentiation history and thermochemical evolution, are left to be inferred from laboratory-based experimental work. Continuing to pursue laboratory-based work, including specific experiments to place tighter constraints on the geochemical evolution of the planet and the testing of new, top-of-the-line geochemical instruments, is crucial to prepare for the future exploration of Mercury.

\subsection{LABORATORY WORK AHEAD OF FUTURE EXPLORATION}

The X-Ray Spectrometer (XRS), Neutron Spectrometer, and Gamma-Ray Spectrometer instruments aboard the MESSENGER spacecraft provided the first detailed chemical analyses of Mercury's surface. Among the many discoveries about Mercury made by MESSENGER, several surprising compositional characteristics of the surface were observed. Recent studies show carbon enrichment as high as $4 \mathrm{wt} \%$ over the local mean in the low reflectance materials excavated from depth, and as much as $2.5 \mathrm{wt} \%$ carbon enrichment in regional deposits associated with the most heavily-cratered terrains. Furthermore, the XRS measured up to $3 \mathrm{wt} \%$ sulfur (S) and less than 2 $\mathrm{wt} \%$ iron $(\mathrm{Fe})$ on Mercury's surface, suggesting the planet's oxygen fugacity $\left(f \mathrm{O}_{2}\right)$, or partial pressure of $\mathrm{O}_{2}$ gas in equilibrium with a magmatic system, is between 2.6 and $7.3 \mathrm{log}$ units below the Iron-Wüstite (IW) buffer (Figure 1). Under such highly reducing conditions, the majority of the Fe available on the planet partitions into the core, leaving behind a silicate mantle that is highly depleted in $\mathrm{FeO}$, although the magnitude of the depletion is still an open question. In fact, estimates of the $\mathrm{FeO}$ abundance in the mantle range from 1.0-2.2 $\mathrm{wt} \%$ on the basis of measured iron abundances from the surface, and from $0.02-0.03 \mathrm{wt} \%$ as informed by melting experiments conducted at an $f \mathrm{O}_{2}$ of 5-6 log units below the IW buffer. Even more enigmatic, Mercury also has a large core and thin mantle compared with the other terrestrial planets in the solar system, with 
the core-mantle boundary at an estimated pressure of only 4-7 GPa. Combined, these constraints have important implications for the constitution, structure, and thermochemical evolution of Mercury and warrant further investigation by laboratory-based studies. (The data above are from [3] and references therein.)

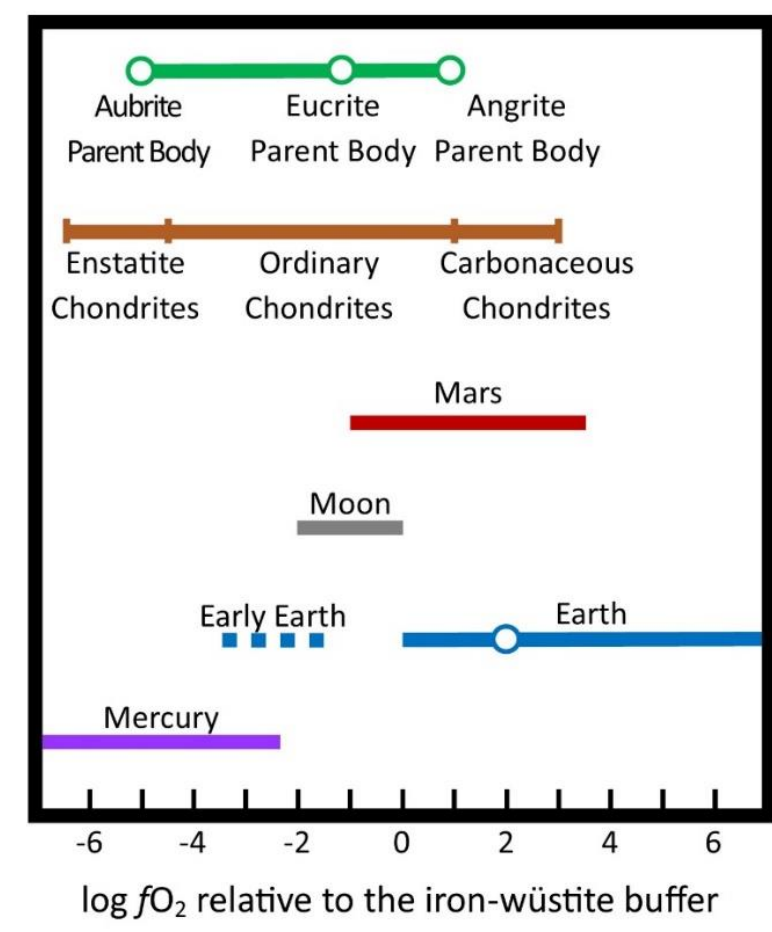

Figure 1. Relative $\mathrm{fO}_{2}$ conditions for some rocky bodies across the solar system. Modified after [4].
Given the high carbon abundances, low oxygen fugacity, and shallow mantle, studies have suggested the presence of a graphite flotation crust as the primary crust on an early mercurian magma ocean [5]. The presence of this graphite flotation crust has major influences on the thermochemical evolution and differentiation of the planet. Laboratory studies focusing on the crystallization of a mercurian magma ocean at varying pressure and temperature conditions are vital to furthering our understanding of this geochemical endmember. In addition, the $f \mathrm{O}_{2}$ plays an important role in the physicochemical properties of a given system, since $f \mathrm{O}_{2}$ has a substantial impact on the partitioning behavior between and solubility of elements in various phases. The reduced nature of planetary bodies like Mercury, the aubrite parent bodies, and some exoplanets have raised many questions regarding the geochemical behavior of typically lithophile elements in magmas at low $\mathrm{fO}_{2}$. Determining the geochemical affinity of major, minor, and trace elements in S-rich, reduced, rocky planetary bodies through experimental and analytical studies will provide crucial insights into the distributions of these elements among metal, sulfide, and silicate phases on bodies like Mercury. These data will fill a critical gap in our understanding of the behavior of elements under reduced conditions in complex geological systems. Furthermore, when compared with the geochemical behavior of elements under more oxidizing conditions, these data will help to constrain models of element distribution and behavior over a wide range of conditions relevant to planetary systems. Finally, understanding elemental behavior at reduced conditions is a prerequisite to characterizing the bulk composition of these planetary bodies, including that of their cores, mantles, and crusts. Therefore, there is a critical need to systematically determine the behavior of elements in the presence of $S$ under reducing conditions, both experimentally and via sample analysis, to understand the fate of elements and the role of $S$ during the differentiation and thermal evolution of reduced bodies in the solar system.

In addition to investigating the geochemical affinity of major, minor, and trace elements, experiments determining the amount of volatiles stored in silicates in Mercury's mantle and partitioned into mercurian magmas are key to understanding the surprising MESSENGER result of high K/Th (a ratio of a volatile element over a refractory element used to indicate the level of volatile depletion of planetary bodies) that implies Mercury is still volatile-rich. The first flyby of MESSENGER revealed widespread pyroclastic deposits and enigmatic hollows (likely formed by volatile sublimation from within rocks exposed to the harsh conditions at Mercury's surface), 
suggesting Mercury had much more volatiles than the Moon. These pyroclastic deposits were found to be depleted in $S$ relative to their surroundings, implying $S$ may be a driver of explosive volcanism on Mercury. However, because $f \mathrm{O}_{2}$ influences the partitioning of many elements, the total volatile inventory of Mercury remains an open question.

Volatile content in silicate minerals like orthopyroxene and olivine will also affect the rheology of the mercurian mantle and the onset of melting. The volatile content of mercurian melts will also influence the magma ocean and magma eruption dynamics of the planet. The identity of volatiles including Sulfur that drove mercurian pyroclastic eruptions is largely unknown, although up to 1.3 wt. \% is required depending on molecular mass [6] and volatile species [7]. Experimental studies that place bounds on the upper limits of each volatile element that could be responsible for pyroclastic volcanism on Mercury will considerably aid our understanding of the planet's geologic history. Experiments at variable pressures could also establish how much juvenile gas is released upon depressurization. We emphasize again, however, that the topics addressed in this section are a small fraction of the geochemical related studies that need to take place over the next decade in preparation for continued successful exploration of Mercury.

\subsection{TESTING GEOCHEMICAL INSTRUMENTS FOR FUTURE EXPLORATION}

In addition to laboratory experiments to characterize the expected composition of the surface, it will be important to test geochemical and mineralogical instruments that may be used in future Mercury exploration. Following the MESSENGER and BepiColombo orbiters, the best next step in Mercury exploration is a landed mission [1]. The mercurian surface materials are highly reduced and volatile-rich, creating unique mineral assemblages not seen anywhere else in the solar system. Geochemical and mineralogical instruments typically flown on planetary missions should be optimized to measure the composition of the mercurian surface, and we must identify which instruments will result in the greatest scientific return from a landed mission. These instruments include X-ray diffractometers, Raman and infrared (IR) spectrometers, Mössbauer spectrometers, evolved gas analyzers, X-ray fluorescence spectrometers (with or without elemental mapping capabilities), and laser-induced breakdown spectrometers. Once the instruments for a nominal payload are identified, they should be matured to flight-readiness. Furthermore, if these instruments require a sample delivery system, the methods of sample delivery should be evaluated (e.g., drill/scoop on a robotic arm, pneumatic sample delivery, etc.). Coupling these investigations with the experimental efforts discussed above will maximally ensure we are prepared to continue to explore the geochemical nature of the Sun's closest planetary neighbor.

\subsection{GEOLOGY RELATED STUDIES}

Fieldwork provides the basis not only for ground-truthing remotely sensed observations, but for acquiring samples of analogue materials that offer direct insight into the chemical and physical properties of rocks on other worlds. Geological laboratory studies include assessment of the mechanical characteristics of rock (e.g., compressibility, fracture density, porosity, permeability, etc.), and employ a wide variety of techniques including conventional microscopy of samples and thin sections (under plane and polarized light), scanning and transmission electron microscopy, Xray diffraction, fluorescence, and tomography, and well as cathodoluminescence and optically stimulated luminescence. But such analyses can also extend to melting and cooling of lava samples and even subjecting rocks to the terrific forces involved in bolide impacts. For Mercury-focused studies, it makes sense to concentrate on volcanic deposits that have been subjected to tectonic deformation, as such rocks most closely reflect the geological processes on the innermost planet 
(including fracturing and by impacts).

\subsection{FIELDWORK AHEAD OF FUTURE EXPLORATION}

Geological processes and the complexity of geological relationships are first and foremost understood by studying rock exposures in the field, and thus the field is often referred to as a geologist's "laboratory." At analogue field sites here on Earth that are host to landforms, environments, and/or processes found in planetary settings, fieldwork both informs and enables planetary geologists to test hypotheses for remote-sensing observations. We therefore emphasize the importance of fieldwork for future planetary exploration and the education of future scientists involved in the analysis and interpretation of planetary geology. The acquisition of observations of geological relationships, geological field mapping, characterization of aeolian landforms, and measurement of tectonic strain can be carried out with standard geological field techniques [e.g., 8], while also making use of emerging technologies such as remotely piloted aerial vehicles.

There is a wealth of landforms, environments, and processes on Mercury with analogue field sites on Earth. Much of Mercury's surface was shaped and modified by basaltic volcanism and protracted tectonic activity [e.g., 9]. The global contractional tectonophysical setting on Mercury, in particular, dictated much of the volcanic and tectonic processes and produced a plethora of faultrelated landforms on the planet [10]. Therefore, geological fieldwork focused on the geology of Mercury should involve the characterization of faulted volcanic rocks, study the causes and manifestations of volcanism in contractional tectonic settings, and investigate the nature of explosive volcanism and its interplay with tectonics. Exemplary field sites of such settings include: (1) the Yakima Fold and Thrust Belt, a series of shortening landforms deforming flood-volcanic rock units of the Columbia Plateau analogous to shortening landforms in Mercury's Borealis Planitia; and (2) the Long Valley Caldera, a $\sim 15 \times 30 \mathrm{~km}$-wide volcanic collapse structure surrounded by a pyroclastic deposit (the Bishop Tuff) that both superposes and is affected by heavily faulted country rock. The Long Valley Caldera and its deposits are analogous to Nathair facula or other pyroclastic vents on Mercury.

\subsection{LABORATORY WORK AHEAD OF FUTURE EXPLORATION}

The Mercury surface likely features a thick layer of regolith-loosely or unconsolidated rock fragments and dust that overlie the stronger, less fractured rock below. Physical processes including meteoroid bombardment, thermal stresses from diurnal insolation variations, and even interactions with solar and cosmic particles can degrade rocks to form regolith. The preponderance of volcanic units on the surface of Mercury suggests that the regolith there would include individual mineral crystals (e.g., olivine, pyroxene, etc.) in addition to bulk rock. Suitable laboratory analogues for Mercury regolith therefore include basaltic lavas that have been heavily fractured and that can be readily ground into smaller fines and dust.

Data from the MESSENGER mission indicate the volcanic units on Mercury to be mainly basaltic [alkali-rich boninites and komatiites: 11]. Although these rocks are associated with high degrees of partial melting (komatiite) or in subduction settings (boninites), representative basaltic samples from any volcanic setting will nonetheless provide useful insights into the mechanical behavior of rocks on Mercury. This holds true for experiments in which rock samples are heated to above the liquidus to generate glassy lava flows. Sample selection for geomechanical laboratory testing should place emphasis on characterization of mineral and crystal sizes, shapes, and orientations, as well as other physical properties such as the presence and orientations of mineral cleavage, the volume fraction and shape of vesicles, material porosity and permeability, etc., since much of the brittle deformation behavior of rocks that can be tested in the laboratory is independent 
of chemical composition.

Rock-mechanical testing in the laboratory is an effective means of enhancing our knowledge of tectonic deformation on planetary bodies [e.g., 12], and may provide useful physical parameters for geodynamic models of Mercury. There is a wide variety of deformation tests that can be carried out that are specific to the physical environment of Mercury. Triaxial compression tests determine strength and elastic properties of intact and fractured materials, and applied to Mercury analogue samples will tell us of how different-aged materials on the planet might respond to stress. Shear tests of types of Mercury-analogue fault gouge materials - fine-grained, clay-like, and usually unconsolidated rock that develops along faults - will inform us of the frictional properties at the interfaces of two blocks of crust and the changes therein during the development of fault zones. These insights, in turn, will play a key role in our understanding of the seismic behavior of, and types of seismicity caused by, faults on Mercury, especially if a seismic instrument is ultimately deployed there [cf. 1].

Furthermore, deformation experiments and characterization in the laboratory of rocks that experienced fracture annealing/healing, or pseudotachylization from impact events have not yet been performed with the specific goal of exploring the strength and deformability of the country rock beneath impact craters or even larger rock masses on heavily cratered bodies such as Mercury. This type of analysis remains a major avenue of work to better understand the mechanical behavior of planetary crusts. The assessment of impact-produced rocks, e.g., suevite, is starting to paint a fuller picture of the mechanical properties of extraterrestrial rocks [13], but such work has yet to further explore target- and impact-related rock types specific to the geology of Mercury.

Mercury's notable 3:2 spin-orbit resonance exposes the planet surface to large thermal differences-as much as $430^{\circ} \mathrm{C}$ during the day to $-180^{\circ} \mathrm{C}$ at night. This thermal environment is likely to affect the deformation properties of rock on the planet, causing thermal stresses that lead to micro-fracturing. The periodic change in temperature probably caused variations of lithospheric strength and depth across the planet [14], and the build-up and release of associated thermal stresses may trigger fatiguing that could play a previously unexplored role for localization and orientation of tectonic structures. Such thermal stresses are known to have fractured boulders observed by the Spirit rover on Mars [15]. Compression tests and X-ray microtomography of samples subjected to cyclical changes in temperatures will therefore help in exploring the role of fatiguing of Mercury's crustal materials from thermal stresses.

Geological field sites and materials on Earth offer readily accessible analogues to understanding the processes and outcomes we see on other worlds. Future studies of Mercury that consider the planet's geological characteristics and materials should encompass, where possible, the lessons learned from centuries of geological work on Earth. Leveraging the considerable resources of geoscience departments and institutes, either through fieldwork, laboratory analyses, or simply the ongoing work to understand fundamental geological problems in general, will ensure that our continued exploration of Mercury is on as strong a footing as possible.

\subsection{SPACE ENVIRONMENT RELATED STUDIES}

Mercury is also unique among the terrestrial planets in having only a tenuous exosphere but a highly dynamic magnetospheric system. This combination leads to an unusually large and unprecedented degree of interplay between the surface and the space environment surrounding the planet. As reflected in both ground-based and MESSENGER observations of the exosphere, micrometeoroid dust impacts play a large role in the generation of the exosphere, with related effects on space weathering of the regolith. Similarly, MESSENGER observations of the magnetospheric plasma revealed a surprising composition of planetary ions, with implications for 
both the sourcing of those ions and their effects upon the regolith when recycled to the surface. Future exploration of the space environment of Mercury and its relationship to the planet's surface will benefit in several ways from a dedicated course of laboratory studies.

\subsection{LABORATORY WORK AHEAD OF FUTURE EXPLORATION}

Ground-based observations revealed the first of many puzzles regarding Mercury's exosphere with the discovery of $\mathrm{Na}$ [16]. The observed column abundances of $\mathrm{Na}$ were inconsistent with the $\mathrm{O}$ abundances inferred from Mariner 10 data, if the two were in stochiometric balance based on the surface chemistry [17]. The abundance of $O$ in the exosphere is itself a point of some contention in that Mariner 10 levels were far higher than those found by MESSENGER [18], with the MESSENGER upper limits pointing to an even greater disparity in the stochiometric balance between $\mathrm{Na}$ and $\mathrm{O}$. These observations suggest that the release processes for $\mathrm{O}$ at Mercury are poorly understood.

Further complicating the O story are the presence of "water-group" ions in the MESSENGER observations by the Fast Imaging Plasma Spectrometer (FIPS) [19]. Owing to the FIPS resolution, these ions could be $\mathrm{O}^{+}, \mathrm{OH}^{+}, \mathrm{H}_{2} \mathrm{O}^{+}$, or a combination thereof. The origin of these water-group ions is intimately connected to the release of not only $\mathrm{O}$ but also water from the surface, perhaps from the polar regions. However, laboratory insights regarding the release of these species at Mercury are lacking, complicating the interpretation of both the FIPS data as well as the exospheric observations that must be analyzed with large-scale Monte Carlo models that depend on laboratory data and theoretical studies as inputs.

Another mystery in the exospheric observations are the high energies associated with the observed $\mathrm{Ca}$ and $\mathrm{Mg}$ atoms [e.g., 20]. Although it is generally agreed that these species derive from meteoroid impact vaporization (MIV), the energies are often much greater than those that should be imparted to the atoms through MIV. This discrepancy has led to the suggestion that the high energies derive from the photodissociation of molecules, with the excess energy over the dissociation threshold leading to the high-energy atoms [20]. Although reasonable, confirmation of this hypothesis relies on laboratory data to identify what species are released during MIV and what those energy distributions are.

Although a number of theoretical studies have been carried out with respect to Mercury's exospheric generation processes, and show promise for resolving some of the mysteries, these findings need to be confirmed through laboratory measurements. For example, work by [21] showed that ions may be released directly to the exosphere through electron-stimulated desorption, which may explain some of the water-group ions. Other work by [22] has shown that photonstimulated desorption of CaS may explain some of the Ca present in Mercury's exosphere. These studies, however, just scratch the surface of what is needed to fully understand the interconnections among the regolith, the dust environment, the magnetospheric plasma, and the neutral exosphere. Further laboratory-based studies that explore a range of processes, temperatures, energies, dust and ion fluxes, and surface compositions are sorely needed to provide ground truth for the theoretical studies and the inputs to the large-scale models of the neutral exosphere and planetary ions in the magnetosphere.

Equally important in understanding how the surface and space environment interact to generate the neutral exosphere and magnetospheric ions is the opposite question: how is the surface changed by the space environment? Underpinning the interpretation of returned orbiter data is a robust understanding of the influence that space weathering has on the physical and chemical evolution of the mercurian regolith. For example, the contribution to space weathering phenomena from micrometeoroid impacts on Mercury is still largely unknown, as is the impact history. When 
examining the contribution of micrometeoroid impacts to space weathering, some geomorphologic similarities can be drawn between the Moon and Mercury, although the conditions of these impacts are rather disparate between the two bodies. Both the flux of impactors (N/year) and their velocities are expected to be much higher on Mercury in comparison to the Moon, with estimates of 5.5-76 times greater flux and a $60 \%$ greater mean impact velocity at the former [23]. With the higher temperature of Mercury's surface, less impact energy is required for phase transformations of surface material into vapor or melt. Combining this with the increased mean impact velocity, a substantially higher rate of agglutinate production is expected for Mercury in comparison to the Moon, which will have a direct consequence on the spectral properties of the surface. The enhancement of past and future mission data thus requires a concerted effort to understand how the mechanism of micrometeoroid impacts plays a role in space weathering under the unique conditions present on Mercury's surface - and can be directly addressed through experimental campaigns employing either impact- and/or laser-based methodologies to simulate bombardment of chemically appropriate starting materials/simulants.

\subsection{CONCLUSIONS AND RECOMMENDATIONS}

To maximize the scientific return of data from current and future exploration efforts of Mercury, it is critical that laboratory- and field-based research be conducted over the coming decade to shed light on the origin and evolution of this geochemical endmember of terrestrial planet formation. Here, we highlight some example open areas of study that will result in extensive knowledge gain in the geochemistry, geology, and space environment of Mercury and support current and future exploration efforts. However, there is a substantial amount of additional laboratory- and field-based research that should be supported over the next ten years to support future Mercury exploration efforts. It is imperative that laboratory- and field-based studies are supported to facilitate the interpretation of data and preparation of future missions to the innermost planet. Importantly, we recommend that the decadal survey consider the critical role of team dynamics, equity, diversity, inclusion, and accessibility in planetary science. We strongly encourage the decadal survey to consider the state of the profession and these issues - not as separable matters, but as critical steps on the pathway to facilitating laboratory- and field-based studies in support of Mercury, our solar system, and beyond.

\subsection{REFERENCES}

[1] Byrne, P.K., et al. (2018) https://bit.ly/2GqsmC2. [2] Vander Kaaden, K.E., et al. (2019) $S S R, 215(49)$. [3] Solomon, S.C., et al. (2018) Cambridge University Press. [4] Anzures, B.A., et al. (2017) LPSC. [5] Vander Kaaden, K.E. and F.M. McCubbin (2015) JGR:P 120(195-209). [6] Kerber, L., et al. (2009) EPSL 285(263-271). [7] Kerber, L., et al. (2011) PSS 59(1895-1909). [8] Coe, A.L. (2010) Wiley-Blackwell. [9] Byrne, P.K., et al. (2018) Cambridge University Press p. 287-323. [10] Byrne, P.K., et al. (2014) Nat. Geosci. 7(301-307). [11] Vander Kaaden, K.E., et al. (2017) Icarus 285(155-168). [12] Heap, M.J et al. (2017) Icarus, 281(103-114). [13] Heap, M.J., et al. (2020) Icarus 349(11387). [14] Williams, J.-P., et al. (2011) JGR 116(E01008). [15] Eppes, M.-C., et al. (2015) Nat. Comm. 6(6712). [16] Potter, A.E. and T.H. Morgan (1985) Science 229(651-653). [17] Hunten, D.M., et al. (1988) The University of Arizona Press p. 562-612. [18] Vervack Jr., R.J., et al. (2016) GRL 43(11545-11551). [19] Zurbuchen, T.H., et al. (2008) Science 321(90-92). [20] Killen, R.M., et al. (2005) Icarus 173(300-311). [21] McLain, J.L., et al. (2011) JGR 116(E03007). [22] Bennett, C.J., et al. (2016) JGR:P 121(137-146). [23] Cintala, M.J. (1992) JGR:P 97(947-973). 